\title{
Investigating the Benefits of Using 3D Camera Technology to Improve Wound Measurements in a Tissue Viability Service: Outcomes of a Pilot Implementation Study
}

\author{
King BM ${ }^{1 \#}$, Doyle $\mathrm{K}^{1 \#}$, Kelley ${ }^{2 \#}$, Taylor $\mathbf{C}^{1 \#}$ and \\ Davis SF $^{2 * * *}$ \\ ${ }^{1}$ Tissue Viability Nurse Consultant, Sheffield Teaching \\ Hospitals, NHS Foundation Trust, Sheffield, England, UK \\ ${ }^{2}$ Research Fellow, Advanced Wellbeing Research Centre, \\ Sheffield Hallam University, Sheffield, England, UK \\ "Contributed Equally to this Work \\ *Corresponding author: Sally Fowler Davis, Work \\ address; 32 Collegiate Crescent, Sheffield S10 2HP tel \\ 0114225 2439. Home Address; 46 Button Hill Sheffield \\ S11 9HH, England, UK
}

Received: July 08, 2021; Accepted: August 31, 2021; Published: September 07, 2021

\begin{abstract}
Sub-optimal experience and outcomes for people with stalled wounds is common. Clinicians have limited methods for reliably and accurately measure wounds. Depth measurement is an important indicator of healing, and digital methods of imaging the wound may offer increased accuracy and enable clinical decision-making.

This study aimed to implement a Panasonic FZ-M1 toughpad with WoundCareLite software version 1.5.0.0, to enable three-dimensional measurements in Tissue Viability (TV) service. Length, width, and depth measurement were compared with usual manual measurement using a paper ruler alongside a 2D photographic image. Statistical analysis included the comparison of wound dimension measures and a presentation of visual healing trajectories over 4 weeks using run-charts.

30 patients were recruited over five weeks (13 female and 17 male), representing $4 \%$ of the usual caseload. Manual measurement and 3D software automatic method demonstrated that the width and depth $3 \mathrm{D}$ auto measures were more accurate than manual measures but depth measures were often wrong thus making volumetric measures inaccurate. Consistent wound size measurement was feasible, and healing trajectories provide a useful means of continuous assessment.
\end{abstract}

Technology guided measurement has potential benefits over manual measurement as a means of more accurately monitoring healing. In this case, depth measurement could not be accurately assessed in practice and further software innovation is indicated to enable outcome measurement in tissue viability services.

Keywords: Tissue Viability; WoundCareLite; 3D

\section{Introduction}

Chronic wound management was estimated to cost the NHS around $3 \%$ of the healthcare budget $[1,2]$, an estimate based predominantly on health care professionals' time used to manage wounds and associated comorbidities [3]. With increase in demand estimated to be at $11 \%$, an estimated 3.7 million patients with a chronic wound in $2017-18$, will cost in the order of $£ 8$ to $£ 9$ billion per annum. Technology implementation associated with personcentered care in policy guidelines, suggests the needs for improved methods for wound assessment [4-6]. A Minimum Data Set (MDS) for wound assessment is recommended [7] with the inclusion of general health assessment, wound assessment parameters, i.e. wound size; the length, width and depth of a wound. Wound photography has been recommended as part of the MDS [8] with images taken on assessment and at a minimum of monthly dressing changes, to monitor progress. This is to enable the clinician to use an objective measure of the size of the wound as indication of healing. In several studies, a reduction of over $30 \%$ in wound size in a four-week period was a predictive indicator of wound healing $[9,10]$. Wound surface area measurement is important as therapeutic decisions may depend on the change of wound surface area over time [11].

Tissue Viability (TV) services in the UK offer specialist advice and care to patients with complex wounds, including pressure ulcers, surgical wounds, and chronic non-healing or so-called stalled wounds. Evidence pertaining to the impact of wound-care teams, on the prevention and management of chronic and non-healing wounds or stalled wounds, does not currently exist [12]. This is in part due to the inability to reliably measure wound dimensions for the purpose of demonstrating a clinical outcome [13].

Measurement of wounds in practice is traditionally undertaken manually with a paper ruler to capture the longest and widest measurements. Depth information is routinely missing from a wound assessment or is estimated and prone to inaccuracy. Subjective assessment also applies to size of wound, and Schultz et al. [14] argued that simple ruler methods could overestimate the surface 
area by $44 \%$. This is in part because the wound is often measured by different nurses at each visit. Previous studies have demonstrated that variation between wound measurements is largely due to differences in subjective identification of the wound edge by clinicians [15]. In some tissue viability services, electronic record keeping includes the use of a standard template with manually collected data, along with a photographic 2D image. Whilst measurement techniques have been refined [16], using a range of technological methods, there remains a significant problem with the accurate measurement for the purpose of recording wound-healing dimensions, and National Institute of Care Excellence (NICE) guidance suggests that improved outcome measurement come with the addition of depth measurement $[17,18]$.

The aim of the study was to pilot the implementation of a 3D digital method of measuring stalled wounds using the WoundCareLite software (GPC solutions) and to investigate the potential benefits over manual measurement as a means of more accurately monitoring healing within a tissue viability services. The comparison of manual and digital methods was compared to establish whether improved accuracy could be achieved.

\section{Methods}

The pilot implementation study design used a non-randomised purposive sampling of 30 patients over a five-week recruitment period that allowed all patients' wounds to be monitored for up to 12 weeks. Sponsored by Sheffield Teaching Hospitals NHS Foundation Trust in Sheffield UK, the nurses within the Sheffield Tissue Viability Service were trained in the use of the camera equipment and in patient recruitment and consenting good practice via 'Good Clinical Practice' (GCP). Funding was obtained from National Institute for Health Research (NIHR) Wound Management Co-Operative, based on free access to loaned PC and software the Panasonic FZ-M1 toughpad with WoundCareLite software version 1.5.0.0. supplied by GPC woundcare (https://www.gpcsl.com/healthcare. html\#woundcare). NHS Ethics (IRAS approval NIHR 225761) was granted for the study, based on patients' verbal informed consent at treatment that was then recorded in the patient's notes. The electronic health record SystmOne was used to maintain patient records, which has the functionality to collate both $2 \mathrm{D}$ images and TV assessment data. And so additional camera images were collected and stored within usual electronic database; SystmOne.

\section{Protocol}

Tissue viability patients were referred to the service from community nursing and General Practice or other referral agency and were identified as potential participants if they had a non-healing wound that was of more than 4 weeks duration. Wounds of any size were included and the aetiology of the wound was not limited to any one type and adult patients' ages $(<18)$ and genders were not exclusion criteria. Following referral, existing triage methods included the use of a 2-D photo image with paper ruler and a standard wound template assessment, both of which were uploaded to an existing electronic health record (SystmOne). All referrals to tissue viability were triaged and treatment planning was then undertaken with the patient and the community nursing team as per usual care; with advice on dressing and specialist wound management.

Patients were identified, recruited, and consented on referral; provided with an information leaflet and the study was explained. All patients were seen either at a wound clinic or in their own home by the specialist TV research nurse using the digital camera and software and with a community nurse using standard ruler-based measurement. Patients' wounds were measured every four weeks + /2 days during a routine dressing change. All patients' wounds were imaged at $0,4,8$ and 12 weeks or until the wound healed. Each timepoint data collection included:

- Manual measurement of length and width of their wound (known as 'manual measurement') and a 2D photographic image.

- $\quad$ A 3D image taken using the Panasonic FZ-M1 toughpad and WoundCareLite software detected the length, width, depth, surface area and volume of the wound. The 3D modelling and data analytics training to use the camera and software was provided to the research nurse by the company (https://www.gpcsl.com).

All 3D images were stored on a single Panasonic laptop and deleted at the end of the study. 2D images were transferred to SystmOne, thus assuring that no images were held by the company.

Images were first taken with the traditional 2D camera by the attending community nurse, along with measurements using a paper ruler. The camera was positioned $50 \mathrm{~cm}$ above the wound at right angles and $2 \mathrm{D}$ images of the wound were taken. The paper ruler was placed to the side of the wound to indicate length then a second image using the paper ruler to indicate width. Immediately after the rulerbased measurements, a 3D image was taken by the tissue viability research nurse. As with the $2 \mathrm{D}$ camera, the touchpad device was held $50 \mathrm{cms}$ from the wound at right angles and an image taken. The WoundCareLite software indicates when it is at the correct distance from the subject and automatically takes the image when the camera is held still. The 3D images included measurement and these data were stored on the touchpad device. Stored images could be re-measured by the research nurse at any point. In the case of the software failing to differentiate tissue at a wound edge, it was also possible to manually outline the wound edge on the image in the WoundCareLite software to guide the detection of the edge of the wound (3D guided). This was to reduce the occurrence of the software incorrectly identifying the edge of the wound.

\section{Data analysis}

Wound dimensions were automatically provided by the WoundCareLite software on 'auto' mode; to obtain wound measurements of length, width, depth, surface area, and volume. The software automatically detected the wound edge on the image. All wound measurements were collated on SystmOne, manually uploaded alongside usual wound monitoring information. This included 3D images and data and the 2D image and ruler-based measurements for all 30 patients and all the available time points.

Data analysis was undertaken by a statistician who initially collated the data and undertook a review of the demographic spread of recruitment and the range of wounds included in the study. Both the $3 \mathrm{D}$ auto measurements and the $3 \mathrm{D}$ guided were compared to the manual measurements using Bland-Altman plots, scatter plots and R2 correlation coefficients. Comparisons (ruler-based vs 3D auto and ruler-based vs 3D guided) assumed a limit of agreement are between $\pm 10 \mathrm{~mm}$ and $\pm 30 \mathrm{~mm}$ for wounds measurements that are 
typically around 50mm. Limits of agreement demonstrate how far apart measurements from the two different methods are likely to be for most individuals. Where the difference is not clinically important, the two methods can be used interchangeably.

\section{Results}

The normal (mean) rate of referral to tissue viability is 150 patients per week. Over a five-week period, a sample group of 30 participants was identified and recruited allowing the service to report that recruitment achieved $4 \%(30 \times 100 / 750)$ of the population of all patients with non-healing wounds referred to tissue viability. Of all potential participants eligible, two were screened out at the point of consent. One candidate's wound differed from the referral description and another candidate decided not to participate but was the only patient approached who declined to take part.

The most frequent wound by anatomical location was on the lower leg $(n=20)$ and the leg ulcer clinic was the most frequently reported location where wound management was provided (14). 11 participants were seen in their own home by community nurses, 4 self-managed their wound after an initial period, and 1 participant was seen by a practice nurse. Postcodes of the patients represented over half the postcodes in Sheffield and, therefore, a representative sample of a city wide demographic (see table 1) and that proportionally the spread of referrals and recruitment represented a representative sample of patients/wounds who were usually referred to the service.

Table 2 shows the number of participants from whom data were collected at the selected time points and also the reasons for missing-data, including 12 healed wounds during the 12 weeks, two participants admitted to hospital, one death, and one participant failed to attend follow up.

\section{Outcomes of 2D and 3D image measurement}

The length and width measurements made using the $3 \mathrm{D}$ auto method are compared to the standard ruler-based measurements

Table 1: Patient Demographics.

\begin{tabular}{|l|c|c|}
\hline Sex(M/F) & Count & $\%$ of Total \\
\hline F & 13 & $43.30 \%$ \\
\hline M & 17 & $56.70 \%$ \\
\hline Wound Diagnosis & Count & $\%$ of Total \\
\hline Abdominal Surgical & 1 & $3.30 \%$ \\
\hline Abscess- I+D & 1 & $3.30 \%$ \\
\hline Amputation Wound & 2 & $6.70 \%$ \\
\hline Burn & 1 & $3.30 \%$ \\
\hline Dehisced Surgical & 1 & $3.30 \%$ \\
\hline Leg Ulcer & 20 & $66.70 \%$ \\
\hline Moisture Lesion & 1 & $3.30 \%$ \\
\hline Pressure Wound & 3 & $10.00 \%$ \\
\hline Care Provider & Count & $\%$ of Total \\
\hline Community Nurse & 11 & $36.70 \%$ \\
\hline Practice Nurse & 1 & $3.30 \%$ \\
\hline Leg Ulcer Clinic & 14 & $46.70 \%$ \\
\hline Self-Management & 4 & $13.30 \%$ \\
\hline
\end{tabular}

Table 2: Data collection over time.

\begin{tabular}{|l|c|c|c|c|}
\hline \multicolumn{1}{|c|}{ No. of Patients } & Baseline & Week 4 & Week 8 & Week 12 \\
\hline Observed & 30 & 22 & 14 & 11 \\
\hline Healed & 0 & 1 & 4 & 7 \\
\hline Hospitalised & 0 & 1 & 1 & 1 \\
\hline Died & 0 & 1 & 1 & 1 \\
\hline DNA & 0 & 4 & 6 & 4 \\
\hline Photographic error & 0 & 1 & 1 & 3 \\
\hline NA - other & 0 & 0 & 3 & 3 \\
\hline Total & 30 & 30 & 30 & 30 \\
\hline
\end{tabular}

using Bland-Altman plots in Figure 1. It is assumed that usual care would involve the nurse identifying the wound size by visual inspection when measurements are $>50 \mathrm{~mm}$ different to the actual size of the wound. In such cases, the nurse would re-measure. Therefore, differences $>50 \mathrm{~mm}$ were removed from the analysis as outliers - these would likely be errors in recording the data into SystmOne and not within the scope of the study. Three outliers were removed for Figure 1 .

The data shows that there is no systematic difference between the standard ruler-based measurements and the $3 \mathrm{D}$ auto measurements. The points with the largest differences are most often the baseline measurements, shown in blue in Figure 1.

The length and width measurements made using the $3 \mathrm{D}$ guided method were compared to the standard ruler-based measurements using Bland-Altman plots in Figure 2, with two outliers removed.

The data again shows that there is no systematic difference between the standard manual ruler-based measurements and the $3 \mathrm{D}$ guided measurements. The 3D guided measurements appear to match the standard manual measurement more closely for length, when compared to the 3D auto in Figure 1(a). Again, many of the baseline (blue) points have the highest difference.

The data shows that there is some agreement between the manual ruler-based measurements and both the $3 \mathrm{D}$ auto and $3 \mathrm{D}$ guided camera-based methods. However, the baseline measurements (blue points in Figures $1 \mathrm{~b}$ and $2 \mathrm{~b}$ ) have the worst agreement.

\section{Depth measurement and analysis}

The standard manual measurement did not permit safe depth measurement of the wound. Therefore, the $3 \mathrm{D}$ auto camera-based methods afford a new opportunity to assess depth and, therefore, four-weekly depth measures were taken using 3D auto and 3D auto guided. However, issues can be identified with the depth measurement if we plot 3D auto against 3D guided (Figure 3).

The points are grouped into three categories in Figure 3; points that show good correlation, points that show a potential systematic error, and points that show random error. You would expect the majority of mases to be the first group, where the manual guide is not required to improve the automatic measurement. The remaining cases should all be in the final group, where the automatic methods have failed and a major correction is applied by the manual guide. However, many points seem to follow systematic behaviour where the guided method is giving a value six times greater than the automatic 


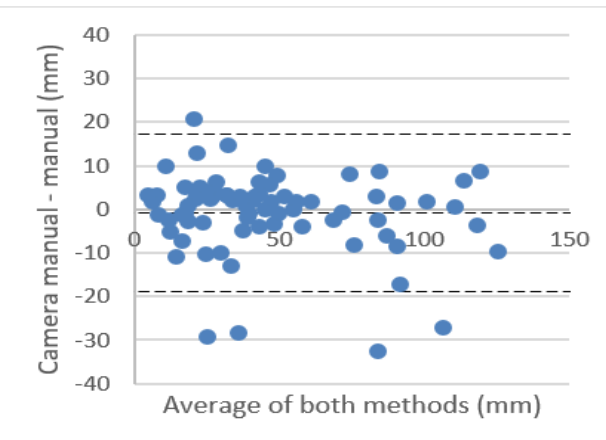

a)

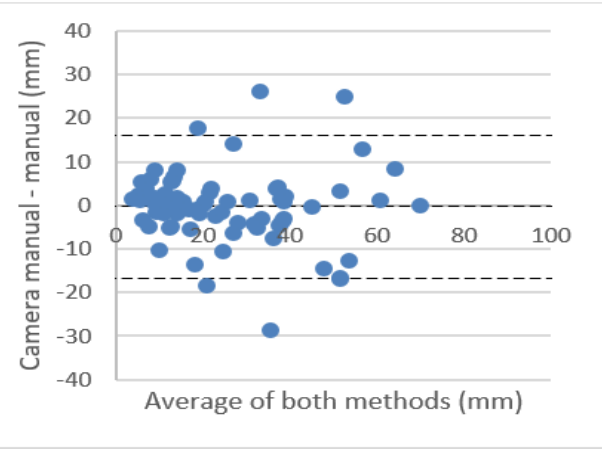

b)

Figure 1: A Bland-Altman plot comparing measurements made by the 3D auto method and the standard ruler-based measurements for a) wound length and b) wound width. The mean difference \pm 1.96 times the standard deviation is shown by the dotted lines. Outliers $>50 \mathrm{~mm}$ difference were omitted.

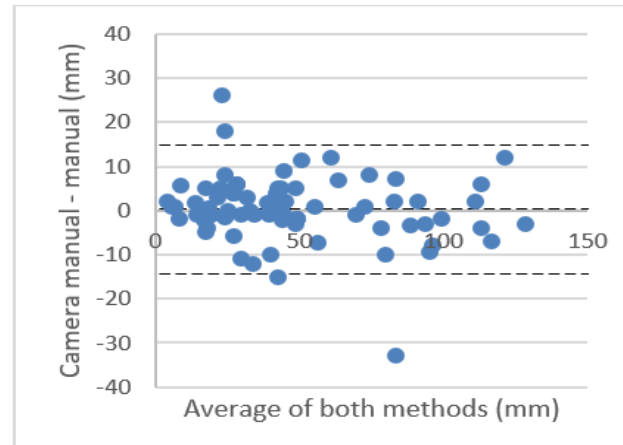

a)

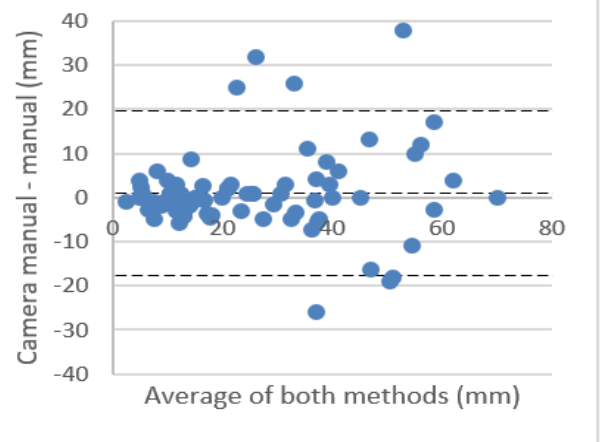

b)

Figure 2: A Bland-Altman plot comparing measurements made by the 3D guided method and the standard ruler-based measurements for a) wound length and b) wound width. The mean difference \pm 1.96 times the standard deviation is shown by the dotted lines. Outliers $>50 \mathrm{~mm}$ difference were omitted.

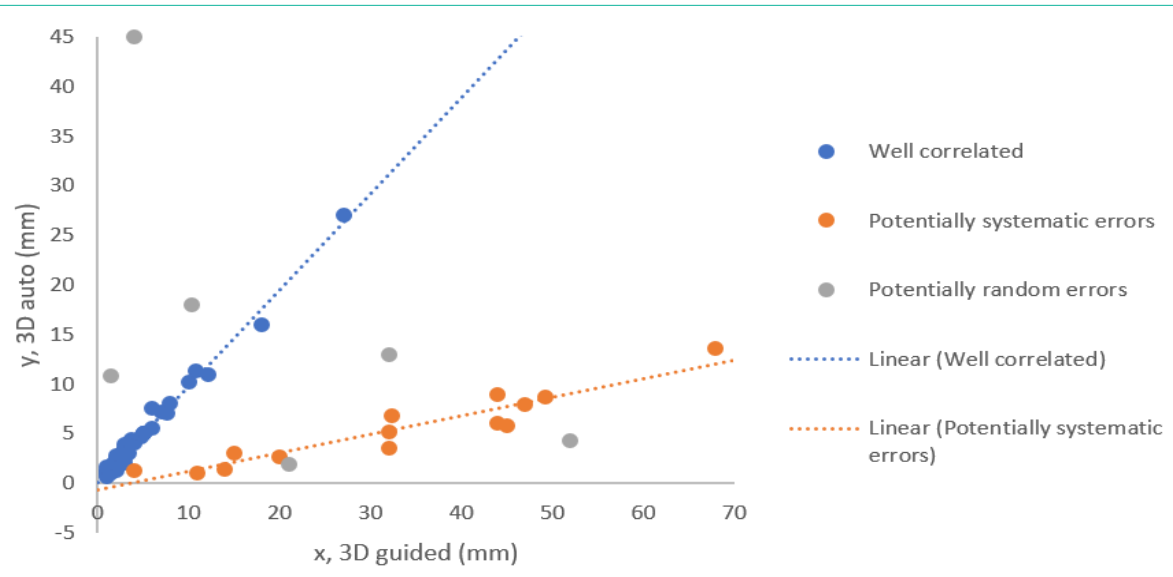

Figure 3: A scatter plot of the depth measurement using the 3D auto method against the depth measurement using the 3D auto guided method. Linear trend lines are shown for the well-correlated points and the points that show a potentially systematic error.

method.

\section{Volumetric measurement}

The surface area measurements from the 3D auto method and the $3 \mathrm{D}$ guided method were in good agreement, had relatively few outliers, and an R2 value of 0.967 . WoundCareLite also calculated volume but $3 \mathrm{D}$ guided volume measurements had similar issues to the depth measurements in Figure 3. This is likely to be because the depth measurements are used to calculate the volume measurements.

\section{Discussion}

This study aimed to pilot the implementation of the touchpad and WoundCareLite as a method to enable length, width and depth 
measurements of non-healing wounds referred to the tissue viability service. This contrasts with testing camera and software devices under laboratory conditions using plastic wound moulds for the validation of data and reliability of the device. The introduction of the touchpad and WoundCareLite software to image wounds was made easier due to the pre-existing experience of using $2 \mathrm{D}$ images for wound assessment and the ability to collect and store images on the electronic record. The formal adoption of measurements, facilitated by the WoundCareLite software, could be an important step in the use of an enhanced system for wound measurement that could be shared via the electronic records SystmOne, which facilitates access to data across community nursing enabling treatment monitoring and review, based on empirical evidence gathered locally or remotely.

The primary goal of a TV service is to provide specialist wound management that results in rapid healing of stalled wounds with maximum functional outcomes and aesthetic results [19]. The study successfully recruitment 30 patients and no adverse outcomes to patients was caused by regular monitoring and increased camera use. The study presents also demonstrates the importance of systematically implementing a technology within a service to assess the added value and benefits to patient care. Wound non-invasive imaging for measurement is a promising advancement in wound assessment but this technology along with others requires further validation [20].

As stated previously, there are recognized anomalies and inconsistencies resulting from paper ruler measurement and also limitations in achieving any accurate, non-invasive depth measure. The study identified that measures of length and width of the wound, calculated by the WoundCareLite software on auto mode were broadly consistent with the manual measurement and the technology-based measurement, for all wounds across time points. The use of imaging was therefore found to be acceptable to patients and staff.

However, there is no gold standard for wound measurement and as each wound is measured, there is an assumed bias based on non-zero dimensions for length, width, and depth that reduces the accuracy of wound size, relative to healing. Similarly, there is no defined tolerance, and each wound is currently measured relative to the healing, based on an overall assessment of the patient. Measures of length, width and depth demonstrate a possible use of the WoundCareLite software as a tool to empirically measure outcome of treatment and wound resolution. It should be noted that volumetric measurement was also taken for the wound, but these measures became unreliable once the depth error was made known.

\section{Wound measurement protocol}

The length and width measurements made using the 3D guided method were compared to the standard manual measurements using paper ruler using Bland-Altman plots (Figures 2 and 3). Ruler method applied consistently can provide reliable wound measurement [21] is therefore regarded as usual practice. The systematic collection of $3 \mathrm{D}$ data in the study re-enforced the importance of systematically using measurement to plot healing [22] with regular assessment, including consistent measurement practices, is clearly better than no measurements being taken [23]. The limits of agreement $(1-3 \mathrm{~cm}$ difference) show that both the $3 \mathrm{D}$ auto and $3 \mathrm{D}$ guided methods cannot be used interchangeably with the standard ruler-based method.

A decrease in wound depth is thought to be an important prognostic indicator of wound healing, as it provides evidence of otherwise imperceptible changes in wound size [7-9]. But studies in burns recognize that the "indeterminate" depth [24] of wounds and healing patterns often defy systematic measurement, even with optical and Laser Doppler imaging [25]. In this case, visual appraisal of depth during clinical assessment can identify the variation in wound healing whereas the technology more simply sought to identify the deepest part of the wound. This continually changed making comparison of data impossible. In addition, the 3D imaging and software measures were prone to a systematic error that was identified only when plotting the data and undertaking statistical analysis. The failing in the software caused the guided method to produce results six times the automatic measurement. Whilst the software can be corrected in time, this demonstrates the importance of piloting technology in practice to ensure that investment in new methods provides a more reliable measurement than usual care and standard methods. Further software or procedural development is required to improve the system and enable depth and volumetric measurement.

The adoption of a digital software to improve the accuracy of wound measurement and therefore plot healing remains an important goal for software developers, perhaps working closely with clinical teams, as was demonstrated in this study. The pilot study resulted in a decision to continue with manual ruler-based methods with $2 \mathrm{D}$ images on SystmOne but also reinforced the importance of accurate continuous measurement by TV nurses; enabling clinical reasoning to assess wound healing. The visual assessment of depth of wound remains important in on-going management, providing a continuous report of the overall deepest part at any time -point. 3D measurement and other smart technologies are an important and developing area for study, particularly in relation to the implementation of new and more effect methods of primary prevention, perhaps identifying wound formation before appearance [26].

\section{Strengths and Limitations}

This study enabled a clinical team to carefully test the use of a $3 \mathrm{D}$ software with the goal of improving and standardizing measurement in a tissue viability service and within the funding was able to test the feasibility of recruiting and retaining patients to a 12 week pilot in which measurement methods could be compared. The study was somewhat limited by a software error that led to false results and reduced confidence in use but demonstrated the importance of systematic piloting of digital methods.

\section{Conclusions}

The use of the 3D imaging and the WoundCareLite software provided comparably accurate measurement for most images and was acceptable to patients within the pilot study. Tissue Viability nurses preferred to use the $3 \mathrm{D}$ guided method (drawing around wound image before taking measures) to confirm the edge of the wound, using clinical judgement to inform decision about the size of the wound but this did not enhance the automatic measurements. The pilot reinforced the importance of visual assessment and checking and confirming the computer-generated metrics in practice and the need for TV nurses to develop capability with technology. Further development of the WoundCareLite software may enable more accurate assessment of depth measurement. As there are no 
better tools to undertake depth measurement safely and accurately, a software solution would be highly advantageous in wound management. Depth and therefore volumetric measurement, based on accurate three-dimensional metrics would enhance clinical decision-making and for use in research.

\section{Declaration}

Author Contributions: BK and SFD undertook the drafting of the paper, based on the content of the final report to the NIHR Wound Management Co-Operative and research results. KD collected data and collated the results, KK collated the data and JK completed the statistical analysis. CT and LF contributed to the set-up of the study, the discussion and supported the final submission amending several drafts.

Acknowledgements: Steering group participation including Kim Drewry Tissue Viability nurse, Tracey Barlow Tissue Viability nurse, Helen Chapman Head of Community Nursing and Lisa Farndon Research lead for the Care Group.

\section{References}

1. Posnett J, Franks PJ. The burden of chronic wounds in the UK. Diabetic Med 2008; 14: S7-85.

2. Guest JF, Ayoub N, Mcllwraith T, Uchegbu I, Gerrish A, Weidlich D, et al. Health economic burden that wounds impose on the National Health Service in the UK. BMJ open. 2015; 5: e009283.

3. Guest JF, Ayoub N, Mcllwraith T, Uchegbu I, Gerrish A, Weidlich D, et al. Health economic burden that wounds impose on the National Health Service in the UK. BMJ open. 2015; 5: e009283.

4. King B, Morton N, Anderson I. Improving patient outcomes: NHS England's workstream for the lower limb. Wounds UK. 2018; 14

5. NHS England. Commissioning for Quality and Innovation (CQUIN). Guidance for 2017-2019.

6. Peate I. A call to action to improve the visibility of research by nursing midwifery and care staff. British Journal of Nursing. 2018; 27: 98-100.

7. Coleman S, Nelson EA, Vowden P, Vowden K, Adderley U, Sunderland L, et al. Development of a generic wound care assessment minimum data set. Journal of tissue viability. 2017; 26: 226-240.

8. Coleman S, Nelson EA, Vowden P, Vowden K, Adderley U, Sunderland L, et al. Development of a generic wound care assessment minimum data set. Journal of tissue viability. $2017 ; 26$ : 226-240.

9. Kantor J, Margolis DJ. A multicentre study of percentage change in venous leg ulcer area as a prognostic index of healing at 24 weeks. British Journal of Dermatology. 2000; 142: 960-964.

10. Sheehan P, Jones $P$, Caselli A, Giurini JM, Veves A. Percent change in wound area of diabetic foot ulcers over a 4-week period is a robust predictor of complete healing in a 12-week prospective trial. Diabetes care. 2003; 26 : 1879-1882.
11. Foltynski $P$. Ways to increase precision and accuracy of wound area measurement using smart devices: Advanced app Planimator. PloS One. 2018; 13: e0192485

12. Moore ZE, Webster J, Samuriwo R. Wound-care teams for preventing and treating pressure ulcers. Cochrane Database of Systematic Reviews. 2015.

13. Keast DH, Bowering CK, Evans AW, Mackean GL, Burrows C, D'souza L. Contents: Measure: A proposed assessment framework for developing best practice recommendations for wound assessment. Wound Repair and Regeneration. 2004; 12: s1-s17.

14. Schultz G, Mozingo D, Romanelli M, Claxton K. Wound healing and TIME: new concepts and scientific applications. Wound Repair and Regeneration. 2005; 13: S1-S11.

15. Terris DD, Woo C, Jarczok MN, $\mathrm{Ho} \mathrm{CH}$. Comparison of in-person and digital photograph assessment of stage III and IV pressure ulcers among veterans with spinal cord injuries. Journal of Rehabilitation Research \& Development. $2011 ; 48$.

16. Langemo DK, Melland H, Hanson D, Olson B, Hunter S, Henly SJ. Twodimensional wound measurement: comparison of 4 techniques. Advances in wound care: the journal for prevention and healing. 1998; 11: 337-343.

17. Leaper D, Burman-Roy S, Palanca A, Cullen K, Worster D, Gautam-Aitken E, et al. Guidelines: prevention and treatment of surgical site infection: summary of NICE guidance. BMJ: British Medical Journal. 2008; 337: 1049-1051.

18. Bell-Syer S, Brady M, Bruce J, Cullum N, Foxlee R, Jull A, et al. Evidencebased wound care in the UK: a response to David Leaper's editorial in International Wound Journal April 20096 (2). International wound journal. 2009; 6: 306-309.

19. Singer AJ, Dagum AB. Current management of acute cutaneous wounds. New England Journal of Medicine. 2008; 359: 1037-1046.

20. Paul DW, Ghassemi P, Ramella-Roman JC, Prindeze NJ, Moffatt LT, Alkhali A, et al. Noninvasive imaging technologies for cutaneous wound assessment: A review. Wound Repair and Regeneration. 2015; 23: 149-162.

21. Wang SC, Anderson JA, Evans R, Woo K, Beland B, Sasseville D, et al. Point-of-care wound visioning technology: Reproducibility and accuracy of a wound measurement app. PloS One. 2017; 12: e0183139.

22. Langemo D, Anderson J, Hanson D, Hunter S, Thompson P. Measuring wound length, width, and area: which technique?. Advances in skin \& wound care. $2008 ; 21$ : $42-45$.

23. Keast DH, Bowering CK, Evans AW, Mackean GL, Burrows C, D'souza L. Contents: MEASURE: A proposed assessment framework for developing best practice recommendations for wound assessment. Wound Repair and Regeneration. 2004; 12: s1-17.

24. Karim AS, Shaum K, Gibson AL. Indeterminate-Depth Burn Injury-Exploring the Uncertainty. Journal of Surgical Research. 2020; 245: 183-197.

25. McGill DJ, Sørensen K, MacKay IR, Taggart I, Watson SB. Assessment of burn depth: a prospective, blinded comparison of laser Doppler imaging and videomicroscopy. Burns. 2007; 33: 833-842.

26. Scalamandré A, Bogie KM. Smart technologies in wound prevention and care. In Innovations and Emerging Technologies in Wound Care. 2020: 225244. Academic Press. 